\title{
PSEUDOCONVEX NON-STEIN DOMAINS IN PRIMARY HOPF SURFACES
}

\author{
CHRISTIAN MIEBACH
}

\begin{abstract}
We describe pseudoconvex non-Stein domains in primary Hopf surfaces using techniques developed by Hirschowitz.
\end{abstract}

\section{INTRODUCTION}

Let $H$ be a primary Hopf surface. In [LY12] Levenberg and Yamaguchi characterize locally pseudoconvex domains $D \subset H$ having smooth real-analytic boundary that are not Stein. In this note we generalize their result to arbitrary pseudoconvex domains using ideas developed by Hirschowitz in [Hir74] and [Hir75]. For the readers' convenience these ideas are reviewed in a slightly generalized form in Section 2. In Section 3 we review the structure of primary Hopf surfaces in order to describe a certain (singular) holomorphic foliation $\mathcal{F}$ of $H$. This allows us to formulate the following Main Theorem, which is proven in Sections 4 and 5.

Main Theorem. Let $D \subset H$ be a pseudoconvex domain. If $D$ is not Stein, then $D$ contains with every point $p \in D$ the topological closure $\bar{F}_{p}$ of the leaf $F \in \mathcal{F}$ passing through $p$.

I would like to thank Karl Oeljeklaus for helpful discussions on the subject of this paper and Stefan Nemirovski for a suggestion on how to prove Lemma 5.3. I am also grateful to Peter Heinzner and the SFB/TR 12 for an invitation to the Ruhr-Universität Bochum where a part of this paper has been written.

\section{A REVIEW OF HiRSCHOWITZ' METHODS}

In this section we present the methods developed by Hirschowitz in [Hir75] in a slightly more general setup.

Let $X$ be a complex manifold with holomorphic tangent bundle $T X \rightarrow X$, and let $\pi: \mathbb{P T X} \rightarrow X$ be the projectivized holomorphic tangent bundle. A continuous function on $X$ is called strictly plurisubharmonic on $X$ if it is everywhere locally the sum of a continuous plurisubharmonic and a smooth strictly plurisubharmonic function. 
Definition 2.1. Let $\varphi \in \mathcal{C}(X)$ be plurisubharmonic. Then we define $S(\varphi)$ to be the set of $[v] \in \mathbb{P T X}$ such that $\varphi$ is in a neighborhood of $\pi[v]$ the sum of a plurisubharmonic function and a smooth function that is strictly plurisubharmonic on any germ of a holomorphic curve defining $[v]$.

Lemma 2.2. Let $\varphi \in \mathcal{C}(X)$ be plurisubharmonic.

(1) The set $S(\varphi)$ is open in $\mathbb{P T X}$.

(2) If $S(\varphi)=\mathbb{P T X}$, then $\varphi$ is strictly plurisubharmonic on $X$.

(3) If $\sum_{k} \varphi_{k}$ converges uniformly on compact subsets of $X$ where $\varphi_{k} \in$ $\mathcal{C}(X)$, then we have $S\left(\sum_{k} \varphi_{k}\right) \supset \bigcup_{k} S\left(\varphi_{k}\right)$.

Proof. This is [Hir75, Proposition 1.3].

For any plurisubharmonic function $\varphi \in \mathcal{C}(X)$ we define $C(\varphi)$ to be

$\mathbb{P T X} \backslash\{[v] \in \mathbb{P T X} ; \varphi$ is smooth around $\pi[v]$ and $\partial \varphi(v) \neq 0\}$

and then set

$$
C(X):=\bigcap_{\substack{\varphi \in \mathcal{C}(X) \\ \text { plurisubharmonic }}} C(\varphi) .
$$

Every set $C(\varphi)$ (and thus $C(X))$ is closed in PTX. The next lemma is a slight generalization of [Hir75, Proposition 1.5].

Lemma 2.3. Let $X$ be a complex manifold and let $\Omega:=X \backslash \pi(C(X))$. Then there exists a plurisubharmonic function $\psi \in \mathcal{C}(X)$ which is strictly plurisubharmonic on $\Omega$.

Proof. Since $\pi: \mathbb{P T X} \rightarrow X$ is proper, the set $\Omega$ is open in $X$. If $\Omega$ is empty, there is nothing to prove. Therefore let us suppose that $\Omega$ is a non-empty open subset of $X$. Consequently, $\mathbb{P T X} \backslash C(X)$ is non-empty.

For every $[v] \in \mathbb{P} T X \backslash C(X)$ we find a plurisubharmonic function $\varphi_{[v]} \in \mathcal{C}^{\infty}(X)$ with $\partial \varphi_{[v]} v \neq 0$. We claim that the function $\psi_{[v]}:=$ $\exp \circ \varphi_{[v]}$ is strictly plurisubharmonic in the direction of $[v]$. To see this, we calculate

$$
\partial \bar{\partial} \psi_{[v]}=e^{\varphi_{[v]}}\left(\partial \varphi_{[v]} \wedge \bar{\partial} \varphi_{[v]}+\partial \bar{\partial} \varphi_{[v]}\right) .
$$

In other words, we obtain $[v] \in S\left(\psi_{[v]}\right)$. Since $X$ has countable topology, we get an open covering

$$
\mathbb{P T X} \backslash C(X) \subseteq \bigcup_{k=1}^{\infty} S\left(\psi_{k}\right) .
$$

It is possible to find $\lambda_{k}>0$ such that $\sum_{k=1}^{\infty} \lambda_{k} \psi_{k}$ converges uniformly on compact subsets of $X$. To prove this, choose a countable exhaustion $X=\bigcup_{j} K_{j}$ by compact sets with $K_{j} \subset \stackrel{\circ}{K}_{j+1}$. For every $j$ there are 
$\lambda_{k, j}>0$ such that

$$
\sum_{k=1}^{\infty} \lambda_{k, j}\left\|\psi_{k}\right\|_{K_{j}}
$$

converges. Since $\left\|\psi_{k}\right\|_{K_{j}} \leq\left\|\psi_{k}\right\|_{K_{j+1}}$ for every $j$, we may suppose that $\lambda_{k, j^{\prime}} \leq \lambda_{k, j}$ for all $j \leq j^{\prime}$. Defining $\lambda_{k}:=\lambda_{k, k}$ and noting that every compact subset $K \subset X$ is contained in $K_{j_{0}}$ for some $j_{0}$, we conclude

$$
\begin{aligned}
\sum_{k=1}^{\infty} \lambda_{k}\left\|\psi_{k}\right\|_{K} & \leq \sum_{k=1}^{\infty} \lambda_{k}\left\|\psi_{k}\right\|_{K_{j_{0}}} \\
& \leq \sum_{k=1}^{j_{0}} \lambda_{k}\left\|\psi_{k}\right\|_{K_{j_{0}}}+\sum_{k=j_{0}+1}^{\infty} \lambda_{k, j_{0}}\left\|\psi_{k}\right\|_{K_{j_{0}}}<\infty
\end{aligned}
$$

which proves the claim. It follows that the limit function $\psi:=\sum_{k} \psi_{k}$ is continuous and satisfies $S(\psi) \supset \bigcup_{k} S\left(\psi_{k}\right) \supset \mathbb{P T X} \backslash C(X)$, hence it is strictly plurisubharmonic on $\Omega$.

In the following we say that a complex manifold $X$ is pseudoconvex if there is a continuous plurisubharmonic exhaustion function $\rho: X \rightarrow \mathbb{R}^{>0}$.

Lemma 2.4. Let $X$ be a pseudoconvex complex manifold and let $\gamma: U \rightarrow$ $X$ be the integral curve of a holomorphic vector field on $X$ where $U$ is a domain in $\mathbb{C}$. If $\gamma^{\prime}(U)$ meets $C(X)$, then $\gamma^{\prime}(U)$ is contained in $C(X)$. If $X$ admits a smooth plurisubharmonic exhaustion function, then $\gamma^{\prime}(U) \subset$ $C(X)$ implies that $\gamma(U)$ is relatively compact in $X$. In particular, in this case we have $U=\mathbb{C}$.

Proof. Let $\xi$ be the holomorphic vector field on $X$ with integral curve $\gamma$ and suppose that $\gamma^{\prime}(0)=\xi\left(x_{0}\right) \in C(X)$. It is enough to show that 0 is an inner point of the set of $t \in U$ with $\gamma^{\prime}(t) \in C(X)$, for then the closed set $\left(\gamma^{\prime}\right)^{-1}(C(X))$ is also open, hence equal to $U$. In other words, we must prove that for every plurisubharmonic function $\varphi \in$ $\mathcal{C}(X)$ smooth in a neighborhood of $x_{t}:=\gamma(t)$ we have $\xi(\varphi)\left(x_{t}\right)=0$ whenever $|t|$ is sufficiently small.

To do this, choose $\alpha \in \mathbb{R}^{>0}$ such that $x_{0}=\gamma\left(t_{0}\right) \in X_{\alpha}:=\{x \in$ $X ; \rho(x)<\alpha\}$ where $\rho$ is a continuous plurisubharmonic exhaustion function of $X$. Let $\Phi^{\xi}$ be the holomorphic local flow of $\xi$. For $|t|$ sufficiently small we have

$$
\Phi_{t}^{\xi}\left(X_{\alpha+1}\right) \supset X_{\alpha} \ni x_{t}:=\Phi_{t}^{\xi}\left(x_{0}\right) .
$$

Since $\Phi_{t}^{\xi}: X_{\alpha} \rightarrow X_{\alpha+1}$ is holomorphic, $\varphi_{t}:=\varphi \circ \Phi_{t}^{\xi}$ is continuous plurisubharmonic on $X_{\alpha}$ and smooth in a neighborhood of $x_{0}$ for each plurisubharmonic function $\varphi \in \mathcal{C}\left(X_{\alpha+1}\right)$ that is smooth in a neighborhood of $x_{t}$. Following the proof of [Hir75, Proposition 1.6] we construct a continuous plurisubharmonic function $\psi_{t}$ on $X$ which 
coincides with $\varphi_{t}$ in a neighborhood of $x_{0}$. Choose $\beta \in \mathbb{R}$ such that $\varphi_{t}\left(x_{0}\right)<\beta<\alpha$ and note that $K:=\rho^{-1}(\beta) \subset X_{\alpha}$ is compact. Then choose a convex increasing function $\chi$ on $\mathbb{R}$ fulfilling

$$
\begin{aligned}
\chi\left(\rho\left(x_{0}\right)\right) & <\varphi_{t}\left(x_{0}\right) \quad \text { and } \\
\chi(\beta) & >\left\|\varphi_{t}\right\|_{K} .
\end{aligned}
$$

Finally, define $\psi_{t}: X \rightarrow \mathbb{R}$ by

$$
\psi_{t}(x):= \begin{cases}\max \left(\varphi_{t}(x), \chi \circ \rho(x)\right) & : \rho(x) \leq \beta \\ \chi \circ \rho(x) & : \rho(x) \geq \beta .\end{cases}
$$

One checks directly that $\psi_{t}$ is continuous plurisubharmonic and coincides with $\varphi_{t}$ in some neighborhood of $x_{0}$. Consequently, we may calculate

$$
\xi \varphi\left(x_{t}\right)=\left.\frac{d}{d s}\right|_{t} \varphi\left(\Phi_{s}^{\tilde{\xi}}\left(x_{0}\right)\right)=\xi \varphi_{t}\left(x_{0}\right)=\xi \psi_{t}\left(x_{0}\right)=0
$$

since $\xi_{x_{0}}=\gamma^{\prime}(0) \in C(X)$. Therefore we see that $\gamma^{\prime}(t) \in C(X)$ for every $t \in U$ sufficiently close to 0 , which proves the first part of the lemma.

If $\rho$ is smooth, then choosing $\varphi=\rho$ in the argument given above, we see that $\gamma(U)$ lies in a fiber of $\rho$, hence is relatively compact.

\section{StATEMENT OF THE MAin TheOrem}

Let us fix $a_{1}, a_{2} \in \mathbb{C}$ such that $0<\left|a_{1}\right| \leq\left|a_{2}\right|<1$. The automorphism $\varphi: \mathbb{C}^{2} \backslash\{0\} \rightarrow \mathbb{C}^{2} \backslash\{0\},\left(z_{1}, z_{2}\right) \mapsto\left(a_{1} z_{1}, a_{2} z_{2}\right)$, generates a free proper $\mathbb{Z}$-action on $\mathbb{C}^{2} \backslash\{0\}$. By definition, the compact complex surface $H_{a}:=\left(\mathbb{C}^{2} \backslash\{0\}\right) / \mathbb{Z}$ for $a=\left(a_{1}, a_{2}\right)$ is a primary Hopf surface. We will write $\left[z_{1}, z_{2}\right]:=\pi\left(z_{1}, z_{2}\right)$ where $\pi: \mathbb{C}^{2} \backslash\{0\} \rightarrow H_{a}$ is the quotient map.

The torus $T=\mathbb{C}^{*} \times \mathbb{C}^{*}$ acts holomorphically on $H_{a}$ with three orbits. More precisely, we have $H_{a}=E_{1} \cup H_{a}^{*} \cup E_{2}$ where $H_{a}^{*}:=$ $\left(\mathbb{C}^{*} \times \mathbb{C}^{*}\right) / \mathbb{Z}$ is the open $T$-orbit, and where $E_{1}:=\left(\mathbb{C}^{*} \times\{0\}\right) / \mathbb{Z}=$ $T \cdot[1,0]$ and $E_{2}:=\left(\{0\} \times \mathbb{C}^{*}\right) / \mathbb{Z}=T \cdot[0,1]$ are elliptic curves.

Note that $H_{a}^{*}$ is a connected Abelian complex Lie group which thus can be represented as $\mathbb{C}^{2} / \Gamma_{3}$ where $\Gamma_{3}$ is a discrete subgroup of rank 3 of $\mathbb{C}^{2}$. The map $p: \mathbb{C}^{2} \rightarrow \mathbb{C}^{2} / \Gamma_{3} \cong H_{a}^{*}$ is the universal covering of $H_{a}^{*}$. Let $V$ be the real span of $\Gamma_{3}$ and set $W:=V \cap i V$. There are two possibilities. Either $p(W)$ is dense in $V / \Gamma_{3} \cong\left(S^{1}\right)^{3}$, or $p(W)$ is closed, hence compact, hence an elliptic curve $E$. In the first case, we have $\mathcal{O}\left(H_{a}^{*}\right)=\mathbb{C}$, i.e., $H_{a}^{*}$ is a Cousin group, while in the second case $H_{a}^{*} \cong \mathbb{C}^{*} \times E$.

For the following result we refer the reader to [BHPV04, Chapter V.18]. 
Proposition 3.1. The open orbit $H_{a}^{*}$ is not Cousin if and only if $a_{1}^{k_{1}}=a_{2}^{k_{2}}$ for some relatively prime $k_{1}, k_{2} \in \mathbb{Z}$.

Remark. If there exist relatively prime integers $k_{1}, k_{2}$ with $a_{1}^{k_{1}}=a_{2}^{k_{2}}$, then we have the elliptic fibration $H_{a} \rightarrow \mathbb{P}_{1},\left[z_{1}, z_{2}\right] \mapsto\left[z_{1}^{k_{1}}: z_{2}^{k_{2}}\right]$. The generic fiber is the elliptic curve $E=\mathbb{C}^{*} /(z \sim c z)$ where $c:=a_{1}^{k_{1}}=$ $a_{2}^{k_{2}}$. Note that for a generic choice of $a=\left(a_{1}, a_{2}\right)$ the open subset $H_{a}^{*}$ is a Cousin group.

Suppose that $H_{a}^{*}$ is Cousin and let $\xi \in \mathfrak{t}$ be the generator of the relatively compact one parameter subgroup $p(W)$. Let $\xi_{H_{a}}$ be the holomorphic vector field induced by the $T$-action on $H_{a}$. One checks directly that $\xi_{H_{a}}$ has no zeros in $H_{a}$, hence defines a holomorphic foliation of $H_{a}$. Note that the open subset $H_{a}^{*}$ is saturated with respect to $\mathcal{F}$ and that the leaves of $\left.\mathcal{F}\right|_{H_{a}^{*}}$ are relatively compact in $H_{a}^{*}$. The closure of a leaf $F \subset H_{a}^{*}$ in $H_{a}^{*}$ is a Levi-flat compact smooth hypersurface. In fact, these Levi-flat hypersurfaces are the fibers of the pluriharmonic function $\left[z_{1}, z_{2}\right] \mapsto \frac{\log \left|z_{1}\right|}{\log \left|a_{1}\right|}-\frac{\log \left|z_{2}\right|}{\log \left|a_{2}\right|}$ defined on $H_{a}^{*}$, see [LY12]. If $H_{a}$ is elliptic, then it is foliated by elliptic curves. Again, $H_{a}^{*}$ is saturated with respect to this foliation and the leaves are compact in $H_{a}^{*}$. This shows that in both cases we obtain a (singular) holomorphic foliation $\mathcal{F}$ of $H_{a}$ such that the leaves of $\left.\mathcal{F}\right|_{H_{a}^{*}}$ are relatively compact in $H_{a}^{*}$.

We now state the main result of this note.

Theorem 3.2. Let $H_{a}$ be a primary Hopf surface and let $D \subset H_{a}$ be a pseudoconvex domain. If $D$ is not Stein, then $D$ contains with every point $p \in D$ the topological closure $\bar{F}_{p}$ of the leaf $F \in \mathcal{F}$ passing through $p$.

Remark. For locally pseudoconvex domains having smooth real-analytic boundary this result has been obtained by Levenberg and Yamaguchi using the theory of $c$-Robin functions, see [LY12].

\section{EXISTENCE OF PLURISUBHARMONIC EXHAUSTIONS}

In this section we will show that every smoothly bounded locally pseudoconvex domain $D \subset H_{a}$ admits a continuous plurisubharmonic exhaustion function. For this we will modify Hirschowitz' proof of [Hir74, Théorème 2.1].

Proposition 4.1. Let $D \subset H_{a}$ be locally pseudoconvex and suppose that neither $E_{1}$ nor $E_{2}$ is a component of $\partial D$. Then $D$ admits a continuous plurisubharmonic exhaustion function.

Remark. The hypothesis of Proposition 4.1 is fulfilled if $D$ is locally pseudoconvex and smoothly bounded. Hence, Theorem 3.2 indeed generalizes the main result of [LY12]. 
Proof. We define $\Omega:=\{(x, \xi) \in D \times \mathfrak{t} ; \exp (\xi) \cdot x \in D\}$. By definition, $\Omega$ is an open subset of $D \times \mathfrak{t}$ containing $D \times\{0\}$. Since $D$ is locally pseudoconvex in $H_{a}$, it follows that $\Omega$ is locally pseudoconvex in $D \times \mathfrak{t}$.

We define the boundary distance $d: D \rightarrow \mathbb{R}^{>0}$ by

$$
d(x):=\sup \left\{r>0 ;\{x\} \times B_{r}(0) \subset \Omega\right\} .
$$

It is elementary to check that $d$ is lower semicontinuous. Since $\Omega$ is locally pseudoconvex in $D \times T$, every point $x \in \bar{D}$ has an open Stein neighborhood $U$ such that $\Omega \cap(U \times \mathfrak{t})$ is pseudoconvex. Due to a result of Lelong, see [Lel68, Theorem 2.4.2], the function $-\log d$ is plurisubharmonic on $U \cap D$ and therefore everywhere on $D$.

Note that $-\log d \equiv-\infty$ if and only if $D$ contains $H_{a}^{*}$. Since neither $E_{1}$ nor $E_{2}$ is a component of $\partial D$, this implies $D=H_{a}$ so that we may exclude this case in the following.

For every $x \in H_{a}$ the orbit map $\mathfrak{t} \rightarrow T \cdot x, \xi \mapsto \exp (\xi) \cdot x$, is open into its image $T \cdot x$. Thus we see that $-\log d(x)$ goes to infinity as $x$ approaches a point in $\partial D$. Since $\partial D$ is compact, this implies that $-\log d$ is an exhaustion.

To end this proof, one verifies directly that $d$ is upper semicontinuous in any point $x \in D$ such that $d(x) \neq \infty$. Therefore, for every constant $C>0$ the map $\sup (C,-\log d)$ is a continuous plurisubharmonic exhaustion of $D$.

Remark. The proof of Proposition 4.1 shows that the polar set given by $\{-\log d=-\infty\}$ is non-empty if and only of $D$ contains $E_{1}$ or $E_{2}$.

Remark. In [DF82], Diederich and Fornæss give an example of a relatively compact pseudoconvex domain in a $\mathbb{P}_{1}$-bundle over a Hopf surface that has smooth real-analytic boundary but does not admit an exhaustion by pseudoconvex subdomains.

\section{PROOF OF THEOREM 3.2}

Let us start by noting the following simple but important observation.

Lemma 5.1. The domain $H_{a}^{*}$ admits a smooth plurisubharmonic exhaustion function. Consequently, if $D \subset H_{a}$ is pseudoconvex, then $D^{*}:=$ $D \cap H_{a}^{*}$ is likewise pseudoconvex.

If $H_{a}^{*}$ is Cousin, this lemma follows from [Hu10, Proposition 2.4]. If $H_{a}^{*}$ is not Cousin, then $H_{a}^{*} \cong \mathbb{C}^{*} \times E$ clearly has a smooth plurisubharmonic exhaustion.

Let $D \subset H_{a}$ be a pseudoconvex domain which is not Stein. If the pseudoconvex domain $D^{*} \subset H_{a}^{*}$ is not Stein, then $D^{*}$ is saturated with respect to the foliation $\left.\mathcal{F}\right|_{H_{a}^{*}}$, see [GMO13, Theorem 3.1]. Since 
the leaves of $\mathcal{F}$ are relatively compact orbits of a one parameter subgroup of $T$, continuity of the action map $T \times H_{a} \rightarrow H_{a}$ implies that $D$ is saturated with respect to $\mathcal{F}$ in this case. Therefore, let us assume that $D \neq D^{*}$ is Stein. We will complete the proof of Theorem 3.2 by showing that then $D$ is Stein as well.

We note first that $D$ cannot contain $E_{1}$ or $E_{2}$ if $D^{*}$ is Stein. Indeed, due to the continuity of the leaves of $\mathcal{F}$ remarked above, if $E_{1}$ was contained in $D$, then some of the relatively compact leaves of $\mathcal{F}$ would lie in $D^{*}$, contradicting the assumption that $D^{*}$ is Stein.

Let us consider the subset $C(D) \subset \mathbb{P T D}$ defined in (2.1) where $\pi: \mathbb{P T D} \rightarrow D$ is the projectivized tangent bundle. The proof of the following lemma relies essentially on the explicit knowledge of the structure of primary Hopf surfaces.

Lemma 5.2. Let $D \subset H_{a}$ be a pseudoconvex domain. If $D^{*}=D \cap H_{a}^{*}$ is Stein, then we have $\pi(C(D)) \subset D^{*} \backslash D$.

Proof. Suppose that $\pi(C(D))$ meets $D^{*}$. Since $H_{a}^{*}$ is an Abelian complex Lie group, it has a biinvariant Haar measure. Therefore, we can apply the usual convolution technique in order to approximate the continuous plurisubharmonic exhaustion of $D$ uniformly on compact subsets by smooth ones. This allows us to apply Lemma 2.4 to prove existence of a complex one parameter subgroup $A$ of $T$ and a point $x \in D^{*}$ such that $A \cdot x$ is relatively compact in $D$. Note that $A \cdot x$ is not relatively compact in $D^{*}$ since the latter is assumed to be Stein.

We claim that that the closure of such a curve $A \cdot x$ in $D$ (and hence $D$ itself) would have to contain $E_{1}$ or $E_{2}$, which then, as noted above, will contradict our assumption that $D^{*}$ is Stein. In order to prove this claim, consider $A=\left\{\left(e^{t z_{1}}, e^{t z_{2}}\right) ; t \in \mathbb{C}\right\}$ where $z=\left(z_{1}, z_{2}\right) \in$ $\mathbb{C}^{2} \backslash\{0\}$. If $z_{1}=0$ or $z_{2}=0$, we see directly that the closure of $A \cdot x$ contains $E_{2}$ or $E_{1}$. Hence, suppose that $z_{1}, z_{2} \neq 0$. If $\frac{z_{1}}{z_{2}} \notin \mathbb{R}$, already the closure of $A$ in $\mathbb{C}^{2} \backslash\{0\}$ contains $\left\{z_{1}=0\right\} \cup\left\{z_{2}=0\right\}$, thus the closure of $A \cdot x$ in $D$ contains $E_{1} \cup E_{2}$ as well in this case. Therefore, we are left to deal with the case $A=\left\{\left(e^{t}, e^{\lambda t}\right) ; t \in \mathbb{C}\right\}$ where $\lambda=\frac{z_{2}}{z_{1}} \in \mathbb{R}$. Consider the smooth map $\pi_{A}: \mathbb{C}^{*} \times \mathbb{C}^{*} \rightarrow \mathbb{R}^{>0}$ defined by $\pi_{A}\left(w_{1}, w_{2}\right):=\frac{\left|w_{1}\right|^{\lambda}}{\left|w_{2}\right|}$. The closure $\bar{A}$ of $A$ in $T=\mathbb{C}^{*} \times \mathbb{C}^{*}$ is contained in the kernel of $\pi_{A}$. Since $\bar{A} \cdot x$ is closed and non-compact in $D^{*}$, we conclude that $\pi_{A}\left(a_{1}, a_{2}\right)=\frac{\left|a_{1}\right|^{\lambda}}{\left|a_{2}\right|}$ is closed and non-compact in $\mathbb{R}^{>0}$; in particular we have $\left|a_{1}\right|^{\lambda} \neq\left|a_{2}\right|$. Now choose $t_{m} \in \mathbb{C}$ such that $a_{1}^{m} e^{t_{m}}=c$ for all $m \in \mathbb{Z}$. It follows that

$$
\left|a_{2}^{m} e^{\lambda t_{m}}\right|=\left(\frac{\left|a_{2}\right|}{\left|a_{1}\right|^{\lambda}}\right)^{m}|c|^{\lambda} .
$$


Since $\frac{\left|a_{2}\right|}{\left|a_{1}\right| \lambda} \neq 1$, we see that $\left(a_{2}^{m} e^{\lambda t_{m}}\right)$ converges to 0 for $m \rightarrow \infty$ or $m \rightarrow-\infty$. This proves again that the closure of $A \cdot x$ in $D$ contains $E_{1}$ or $E_{2}$.

Combining the Lemmas 5.2 and 2.3, we see that there exists a plurisubharmonic function $\varphi$ on $D$ which is strictly plurisubharmonic on $D^{*}$.

Remark. Due to [Ri68] (see the formulation given in [Dem12, Chapter I.5.E]), we may assume without loss of generality that $\varphi$ is smooth on $D^{*}$.

As we have noted above, $D$ cannot contain $E_{1}$ or $E_{2}$, so that $D \cap$ $E_{1}$ and $D \cap E_{2}$ are closed Stein submanifolds of $D$. Therefore, the following lemma implies that $D$ is Stein, which then completes the proof of Theorem 3.2

Lemma 5.3. Let $X$ be a connected complex manifold endowed with a plurisubharmonic exhaustion $\varphi$. Suppose that there exists a closed Stein submanifold $A$ of $X$ with at most finitely many connected components such that $\varphi$ is strictly plurisubharmonic on $X \backslash A$. Then $X$ is Stein.

Proof. For $\alpha \in \mathbb{R}$ write $X_{\alpha}:=\{\varphi<\alpha\}$. In the first step we will show that $X_{n}$ is Stein for all $n \geq 1$. Since $A$ is Stein, every $A_{n}:=A \cap X_{n}$ is a closed Stein submanifold of $X_{n}$.

Due to [Siu77], we find an open Stein neighborhood $U_{n+1}$ of $A_{n+1}$ in $X_{n+1}$. Consequently, there exists a strictly plurisubharmonic exhaustion function $\psi_{n+1}$ on $U_{n+1}$. Let us choose a relatively compact open neighborhood $V_{n}$ of $A_{n}$ in $U_{n+1}$ as well as a cutoff function $\chi_{n+1}$ which is identically 1 on $V_{n}$ and which vanishes near $\partial U_{n+1}$. Then $\chi_{n+1} \psi_{n+1}: X \rightarrow \mathbb{R}$ is strictly plurisubharmonic in a neighborhood of $A_{n}$ and its Levi form is uniformly bounded from below on $X_{n}$. Moreover, let $\rho_{n}$ be a plurisubharmonic exhaustion of $X_{n}$ which is strictly plurisubharmonic and smooth on $X_{n} \backslash A_{n}$. Then, for $k$ sufficiently large the function $\left.\chi_{n+1} \psi_{n+1}\right|_{X_{n}}+k e^{\rho_{n}}$ is a smooth strictly plurisubharmonic exhaustion function of $X_{n}$, proving that $X_{n}$ is Stein.

Since we know now that $X_{n}$ is Stein, we can apply [Nar62, Corollary 1] which implies that $X_{n-1}$ is Runge in $X_{n}$ for every $n \geq 2$. Therefore $X=\bigcup_{n \geq 1} X_{n}$ is a Runge exhaustion of $X$ by relatively compact Stein open subsets, hence $X$ is Stein, see [GuRo65, Theorem VII.A.10].

\section{REFERENCES}

[BHPV04] Wolf P. Barth, Klaus Hulek, Chris A. M. Peters, and Antonius Van de Ven, Compact complex surfaces, second ed., Ergebnisse der Mathematik und ihrer Grenzgebiete. 3. Folge. A Series of Modern Surveys in Mathematics, vol. 4, Springer-Verlag, Berlin, 2004. 
[Dem12] Jean-Pierre Demailly, Complex Analytic and Differential Geometry, at www-fourier.ujf-grenoble.fr/ demailly/manuscripts/agbook.pdf, version of June 21, 2012.

[DF82] Klas Diederich and John Erik Fornæss, A smooth pseudoconvex domain without pseudoconvex exhaustion, Manuscripta Math. 39 (1982), no. 1, 119-123.

[GMO13] Bruce Gilligan, Christian Miebach, and Karl Oeljeklaus, Pseudoconvex domains spread over complex homogeneous manifolds, Manuscripta Math. 142 (2013), no. 1-2, 35-59.

[GuRo65] Robert C. Gunning and Hugo Rossi, Analytic functions of several complex variables, Prentice-Hall, Inc., Englewood Cliffs, N.J., 1965.

[Hir74] André Hirschowitz, Pseudoconvexité au-dessus d'espaces plus ou moins homogènes, Invent. Math. 26 (1974), 303-322.

[Hir75] Le problème de Lévi pour les espaces homogènes, Bull. Soc. Math. France 103 (1975), no. 2, 191-201.

[Hu10] Alan T. Huckleberry, Remarks on homogeneous complex manifolds satisfying Levi conditions, Boll. Unione Mat. Ital. (9) 3 (2010), no. 1, 1-23.

[Lel68] Pierre Lelong, Fonctionnelles analytiques et fonctions entières ( $n$ variables), Les Presses de l'Université de Montréal, Montreal, Que., 1968, Séminaire de Mathématiques Supérieures, No. 13 (Été, 1967).

[LY12] Norman Levenberg and Hiroshi Yamaguchi, Pseudoconvex domains in the Hopf surface, arXiv:1205.3346 (2012).

[Nar62] Raghavan Narasimhan, The Levi problem for complex spaces. II, Math. Ann. 146 (1962), 195-216.

[Ri68] Rolf Richberg, Stetige streng pseudokonvexe Funktionen, Math. Ann. 175 (1968), 257-286.

[Siu77] Yum Tong Siu, Every Stein subvariety admits a Stein neighborhood, Invent. Math. 38 (1976/77), no. 1, 89-100.

Laboratoire de Mathématiques Pures et Appliquées, CNRS-FR 2956, Université du LitToral Côte d’Opale, 50, RUE F. Buisson, 62228 Calais CEDEX, FRANCE

E-mail address: miebach@lmpa.univ-littoral.fr 